\title{
Curvature sensor using a highly birefringent photonic crystal fiber with two asymmetric hole regions in a Sagnac interferometer
}

\author{
Orlando Frazão, ${ }^{1, *}$ José M. Baptista,, ${ }^{1,2}$ José L. Santos, ${ }^{1,3}$ and Philippe Roy ${ }^{4}$ \\ ${ }^{1}$ INESC Porto - Instituto de Engenharia de Sistemas e Computadores do Porto, \\ Rua do Campo Alegre 687, 4169-007 Porto, Portugal \\ ${ }^{2}$ Departamento de Matemática e Engenharias, Universidade da Madeira, Campus da Penteada, 9000-390 Funchal, Portugal \\ ${ }^{3}$ Departamento de Física da Faculdade de Ciências da Universidade do Porto, Rua do Campo Alegre 687, \\ 4169-007 Porto, Portugal
}

${ }^{4}$ Xlim, Photonics Department, UMR 6172 CNRS University of Limoges, 123 Avenue Albert Thomas, 87060 Limoges, France

*Corresponding author: ofrazao@inescporto.pt

Received 22 January 2008; revised 29 March 2008; accepted 30 March 2008;

posted 4 April 2008 (Doc. ID 91971); published 30 April 2008

\begin{abstract}
A curvature sensor based on a highly birefringent (Hi-Bi) photonic crystal fiber inserted into a Sagnac interferometer is demonstrated. For this purpose, a novel Hi-Bi photonic crystal fiber was designed and fabricated. Half of the microstructured region of the photonic crystal fiber was composed by large diameter holes, while the other half contained small diameter holes. Because of this geometry, the fiber core was shifted from the center and high birefringence appears in the optical fiber. Curvature was applied for three different fiber directions for a range of $0.6-5 \mathrm{~m}^{-1}$. Temperature and longitudinal strain was also characterized for constant curvature. The configuration showed insensitivity to these two physical parameters. (C) 2008 Optical Society of America
\end{abstract}

OCIS codes: $\quad 060.0060,060.2370,060.4005$.

\section{Introduction}

Curvature is an important physical parameter that needs to be monitored in some areas of engineering. In optical fiber sensing, several configurations have been proposed, comprehending solutions that require Bragg gratings or long period gratings. Etched fiber Bragg gratings [1], Bragg gratings written in $D$-type fiber [2], or in multicore fiber [3] are some examples of sensors using Bragg gratings. The first configuration is limited by the fragility of the optical fiber resulting from the chemical etching. On the other hand, writing a Bragg grating in $D$-shape fiber is difficult due to the fiber geometry. At the same time, splicing multicore fibers with standard fibers may pose additional pro-

0003-6935/08/132520-04\$15.00/0

(C) 2008 Optical Society of America blems. Examples of using long period grating are based on either cladding mode resonance or resonant mode splitting [4]. This former solution is very sensitive to several physical parameters, in particular, its cross sensitivity to temperature [5].

An alternative solution consists of using a two-mode fiber as a curvature sensor. It is based on the frequency-shift interaction between two modes in a two-mode fiber with an off-center core. For this case, the fiber is bent; a tension or compression field appears due to the asymmetry induced by the off-center core [6]. An improved version of a two-mode fiber optic curvature sensor includes temperature and strain compensation [7]. Recently, an all-fiber curvature sensor using a two-core photonic crystal fiber as a sensing element was presented [8]. The photonic crystal fiber (PCF) section acts as a two-beam interferometer and, when the PCF is bent, the phase difference changes. 
The same research group proposed a two-dimensional curvature sensor using a multicore PCF [9].

Sensing solutions integrating Hubby fibers in a Sagnac interferometer are of great interest for sensing applications, namely, as sensor elements for strain [10], temperature [11], and displacement [12]. This interferometer consists of an optical coupler where the $3 \mathrm{~dB}$ coupler splits the input light into two counterpropagating beams, which will then cross the loop. They propagate through the Hi-Bi fiber with different velocity. Meanwhile, their own polarization direction varies and the two beams will interfere at the output port. Recently, a Sagnac interferometer containing PCF was proposed as a temperature-independent strain sensor [13].

Here, we propose a curvature sensor based on a novel Hi-Bi PCF with asymmetric holes inserted into a Sagnac interferometer. Because of these fiber properties and through an optimization of fiber direction in which curvature was applied, the group birefringence is modified. The sensing configuration was also characterized for temperature and for longitudinal strain.

\section{Experimental Results}

The novel Hi-Bi PCF was manufactured with pure silica. The Hi-Bi PCF has a $250 \mu \mathrm{m}$ cladding diameter and a $5 \mu \mathrm{m}$ core size. Half of the microstructured region of the photonic crystal fiber was made of holes with a large diameter $(d \sim 3.3 \mu \mathrm{m})$ while the other half contained holes with a smaller diameter $(d \sim 1.6 \mu \mathrm{m})$. The $d / \Lambda$ ratio between the holes' diameters and the pitch was $\sim 0.6$ and $\sim 0.3$, respectively. Because of the fabrication process, the different pressure obtained in the holes created an asymmetric geometry. Then, the fiber core was shifted from the center and a high group birefringence of $3.7 \times 10^{-4}$ was formed in the Hi-Bi optical fiber. Figure 1 presents an image of the cross section of the novel Hi-Bi PCF.

Figure 1 also illustrates the experimental setup, which consists of an optical broadband source with a $1550 \mathrm{~nm}$ central wavelength and a $100 \mathrm{~nm}$ spectral bandwidth. The Sagnac interferometer is made of a $3 \mathrm{~dB}$ coupler and a polarization controller device for interferometer optimization. The length of $\mathrm{Hi}-\mathrm{Bi}$ $\mathrm{PCF}$ used in the Sagnac interferometer was $375 \mathrm{~mm}$. The spectral response is observed using an optical spectrum analyzer (OSA) with a $0.05 \mathrm{~nm}$ resolution. The splice between the Hi-Bi PCF and the SMF 28 was made using a conventional splicing machine. The losses of the sensing head are approximately $15 \mathrm{~dB}$ and include the losses of the Hi-Bi PCF and the two splices between the PCF and the standard single mode fiber. The sensor curvature is given by $\left.1 / r=2 h /\left(h^{2}+L^{2}\right)\right)$, where $h$ is the bending displacement at the center of the Hi-Bi PCF section; $r$ is the curvature radius, and $L$ is the half-distance between the edges of the two clamps [14]. Before characterizing the curvature sensor, the $\mathrm{Hi}-\mathrm{Bi} \mathrm{PCF}$ direction was chosen. The best direction for curvature sensing is with the larger holes in the lower region and the smaller holes in the upper region. In this case, the core is located in the compressed region and the group birefringence is expected to increase.

Figure 2 shows the transmission spectra of the HiBi fiber loop mirror within an $\sim 17 \mathrm{~nm}$ spectral band. A range of curvatures from 0.6 to $5.4 \mathrm{~m}^{-1}$ was applied for three different fiber directions. First [Fig. 2(a)], the large holes are in the lower region and the core is under compression, resulting in a path length decrease and an increase of the birefringence. For this case, the increase of the birefringence is the dominant effect, causing the wavelength response to shift toward longer wavelengths. In the second case [Fig. 2(b)], the small holes are in the upper region and the core is in tension. For this situation, the birefringence remains constant and wavelength response shifts also toward longer wavelengths due to the increase of the optical path length. However, for this case, the sensor is also sensitive to longitudinal strain. Finally [Fig. 2(c)], the core is in the center while the large holes are in the right region and the small holes are in the left region. In the last case, the interferometer is insensitive to curvature because the core is in a neutral zone relative to the curvature.

For calibration purposes, the sensing head was sequentially bent according to a set of known bending radii $(r)$ in the range of 0.2 to $1.5 \mathrm{~m}$. The chosen measurement parameter was the group birefringence $(\beta)$ given by $\beta=\lambda^{2} / \Delta \lambda L$, where $\lambda$ is the central wavelength operation, $\Delta \lambda$ is the spectral width of the interferometer (values obtained by the OSA), and $L$ is the length of the Hi-Bi PCF section. The results obtained are shown in Fig. 3 . For the bending radius
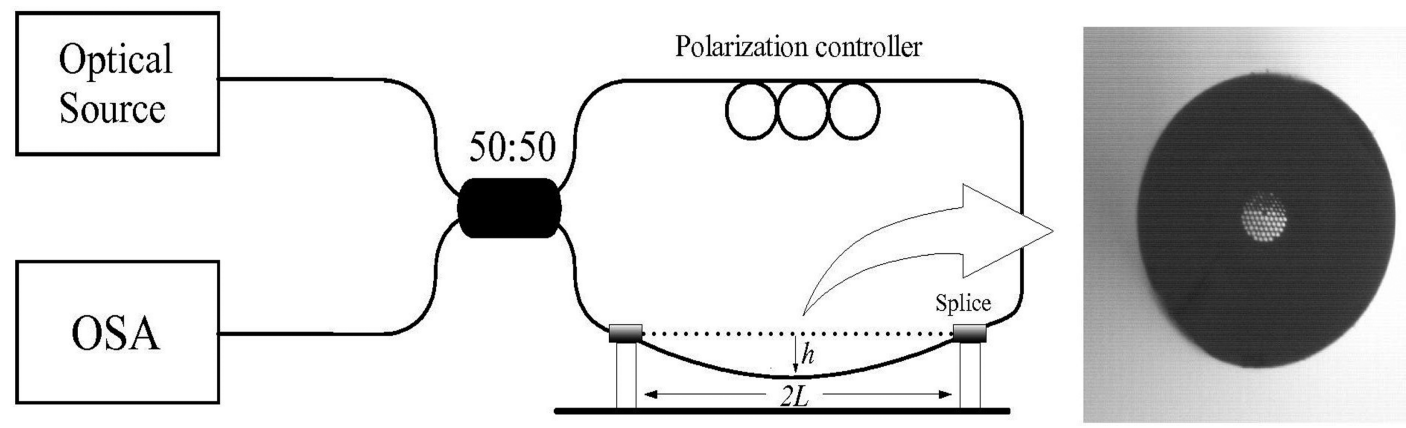

Fig. 1. Experimental setup of the Sagnac interferometer with an image of the novel Hi-Bi PCF showing the two asymmetric hole regions 

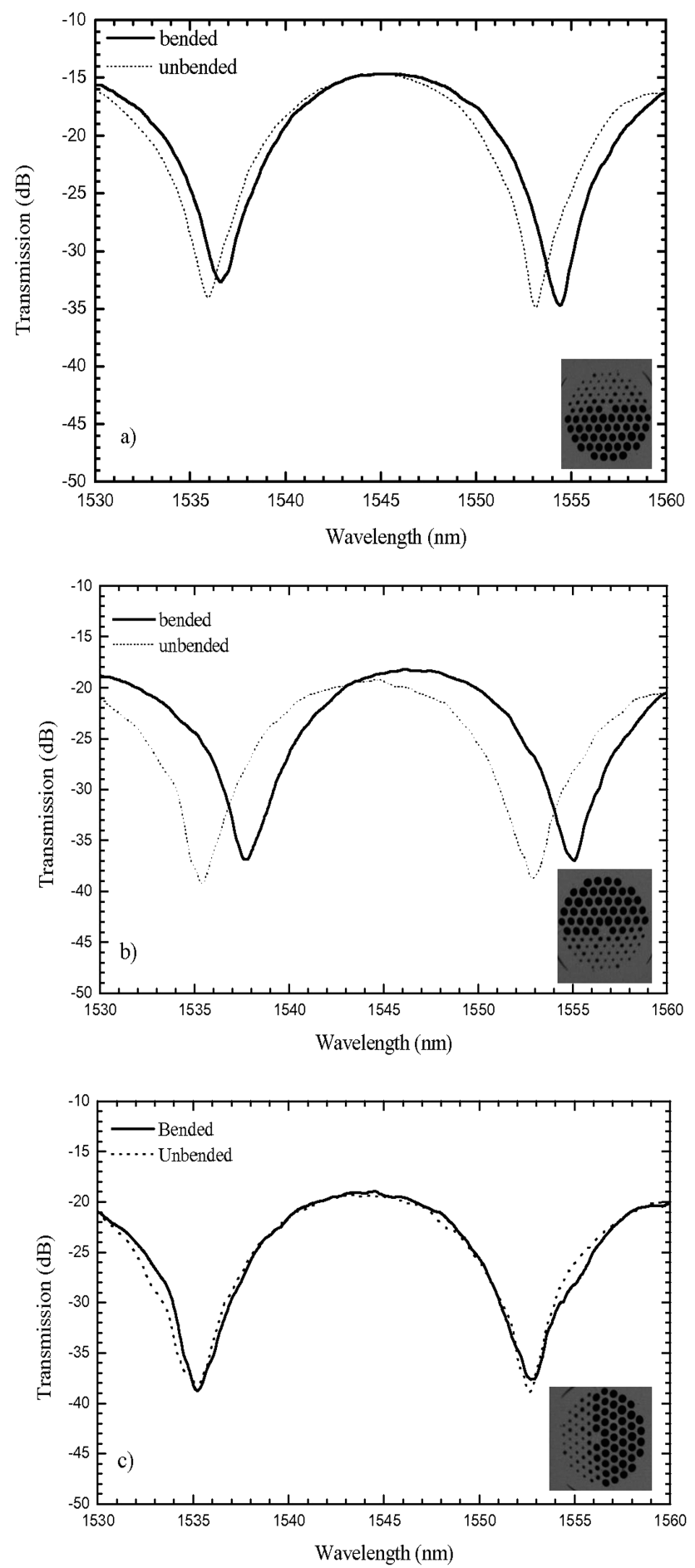

Fig. 2. Spectral response of the Sagnac interferometer for three different curvature directions: a) core in compression, b) core in tension, and c) core in neutral line.

range previously mentioned, the birefringence changed $0.19 \times 10^{-4}$, while the sensor's coefficient sensitivity was obtained through a linear fit and corresponds to $(1.39 \pm 0.07) \times 10^{-5} 1 / \mathrm{m}$. Figure 4 presents the same result as a function of curvature $(1 / r)$.

Figure 5 shows the response of the Sagnac interferometer for two different constant curvatures when temperature was applied. The response of the Sag-

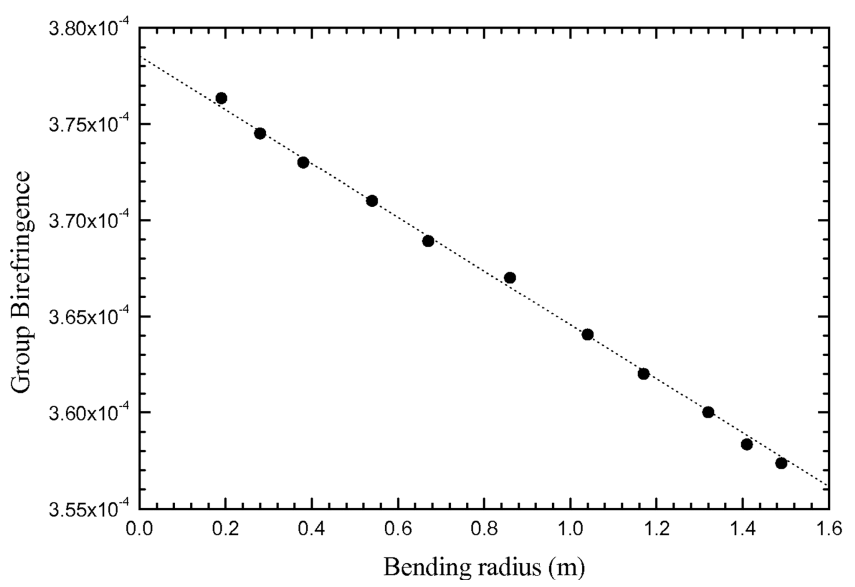

Fig. 3. Response of the Sagnac interferometer as a function of the bending radius.

nac interferometer as a function of the longitudinal strain is shown in Fig. 6 (the Hi-Bi PCF was horizontally stretched. Therefore, no curvature was applied). As can be observed, the two responses present very low sensitivity to both physical parameters. These results were expected because the group birefringence depends only on the Hi-Bi fiber geometry, which remained constant when both physical parameters were applied [15-17].

\section{Conclusions}

Summarizing, a novel Hi-Bi PCF inserted in a Sagnac interferometer was presented and demonstrated as a curvature sensor. This Hi-Bi PCF is made of two regions exhibiting two different radii of holes, which surround a pure silica core. The curvature sensor has maximum sensitivity when the large holes are in the down direction, thus creating compression in the core region. Because of the characteristics of the pure silica and the measurement technique used (group birefringence), the sensor is insensitive to temperature and longitudinal strain. The sensor presented in this article is an alternative solution for curvature

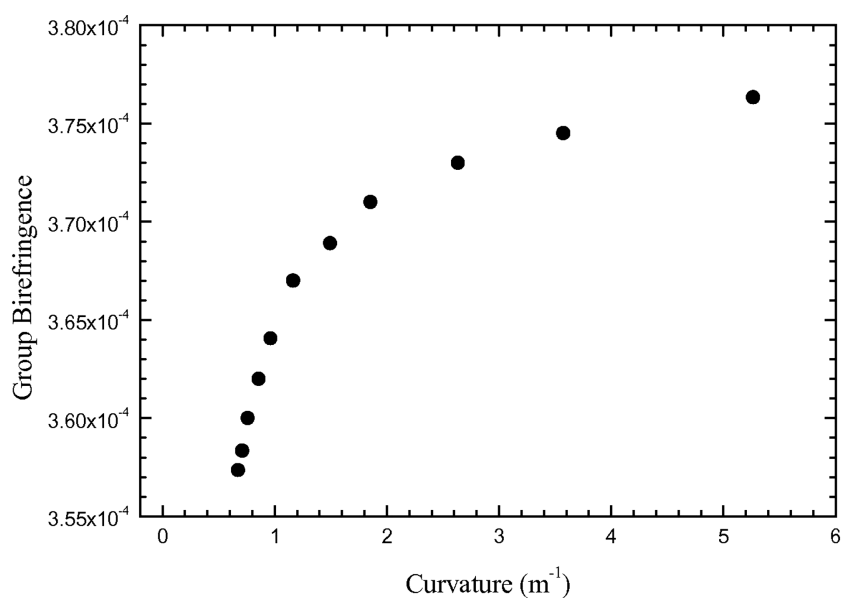

Fig. 4. Response of the Sagnac interferometer as a function of curvature (the inverse of bending radius). 


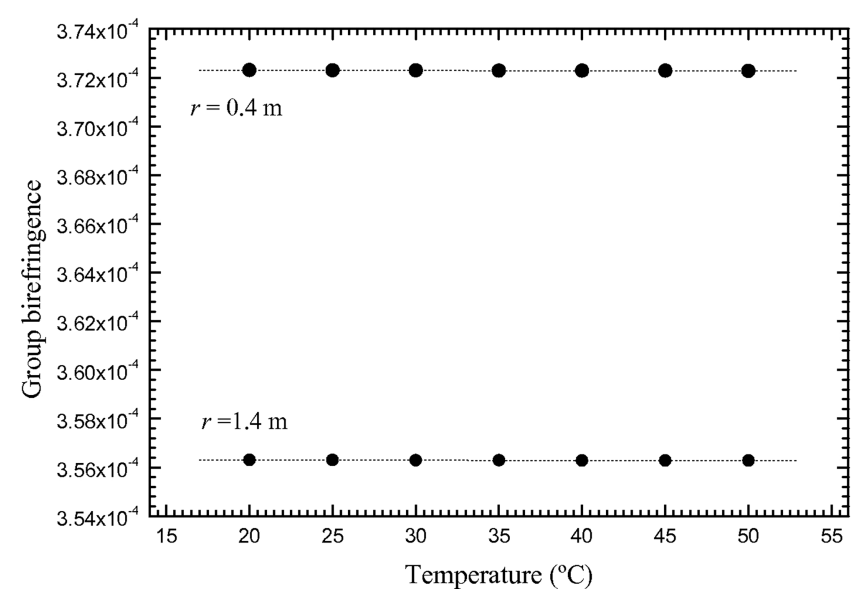

Fig. 5. Response of the Sagnac interferometer when the temperature is applied, for two bending radii $(r=0.4 \mathrm{~m}$ and $r=1.4 \mathrm{~m})$.

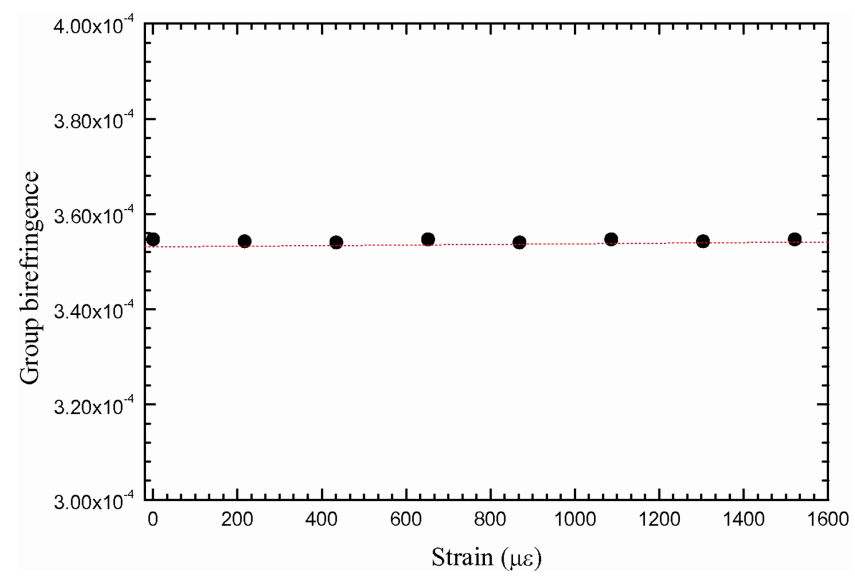

Fig. 6. (Color online) Response of the Sagnac interferometer when longitudinal strain is applied (the fiber was horizontally stretched. Therefore, no curvature was applied).

monitoring in specific applications in some areas of engineering.

This work was supported by the STSM 16-Novel Microstructured Hi-Bi Fibre for Optical Sensing of the COST 299-Optical Fibres for New Challenges Facing the Information Society

\section{References}

1. Y-S. Yu, Z-Y. Zhao, Z-C. Zhuo, W. Zheng, Y. Qian, and Y-S. Zhang, "Bend sensor using an embedded etched fiber Bragg grating," Microwave Opt. Technol. Lett. 43, 414-417 (2004).

2. F. M. Araújo, L. A. Ferreira, J. L. Santos, and F. Farahi, “Temperature and strain insensitive bending measurements with
D-type fiber Bragg gratings," Meas. Sci. Technol. 12, 829833 (2001).

3. M. J. Gander, W. N. Macpherson, R. McBride, J. D. C. Jones, L. Zhang, I. Bennion, P. M. Blanchard, J. G. Burnett, and A. H. Greenaway, "Bend measurement using Bragg gratings in multicore fiber," Electron. Lett. 36, 120-121 (2000).

4. V. Bhatia and A. M. Vengsarkar, "Optical fiber long-period grating sensors," Opt. Lett. 21, 692-694 (1996)

5. O. Frazão, F. M. Araújo, L. A. Ferreira, and J. L. Santos, "Applications of fiber optic grating technology to multi-parameter measurement," Fiber Integr. Opt. 24, 227-244 (2005).

6. O. Lisbôa and C. K. Jen, "An optical-fiber bending sensor using two-mode fibers with an off-center core," Smart Mater. Struct. 3, 164-170 (1994).

7. C. E. Covington, J. Blake, and S. L. A. Carrara, "Two-mode fiber-optic bending sensor with temperature and strain compensation," Opt. Lett. 19, 676-678 (1994).

8. W. N. MacPherson, M. J. Gander, R. McBride, J. D. C. Jones, P. M. Blanchard, J. G. Burnett, A. H. Greenaway, B. Mangan, T. A. Birks, J. C. Knight, and P. St. J. Russell, "Remotely address optical fiber curvature sensor using multicore photonic crystal fiber," Opt. Commun. 193, 97-104 (2001).

9. P. M. Blanchard, J. G. Burnett, G. R. G. Erry, A. H. Greenaway, P. Harrison, B. Mangan, J. C. Knight, P. St. J. Russell, M. J. Gander, R. McBride, and J. D. C. Jones, "Two-dimensional bend sensing with a single, multicore optical fiber," Smart Mater. Struct. 9, 132-140 (2000).

10. M. Campbell, G. Zheng, A. S. Holmes-Smith, and P. A. Wallace, "A frequency-modulated continuous wave birefringent fiber optic stain based on a Sagnac ring configuration," Meas. Sci. Technol. 10, 218-224 (1999).

11. A. N. Starodumov, L. A. Zenteno, D. Monzon, and E. De la Rosa, "Fiber Sagnac interferometer temperature sensor," Appl. Phys. Lett. 70, 19-21 (1997).

12. Y. Liu, B. Liu, X. Feng, W. Zhang, G. Zhou, S. Yuan, G. Kai, and $\mathrm{X}$. Dong, "High-birefringence fiber loop mirrors and their applications as sensors," Appl. Opt. 44, 2382-2390 (2005).

13. O. Frazão, J. M. Baptista, and J. L. Santos, "Temperatureindependent strain sensor based on a Hi-Bi photonic crystal fiber loop mirror," IEEE J Sensors 7, 1453-1455 (2007).

14. W. Du, H.-Y. Tam, M. S.Y. Liu, and X. Tao, "Long-period fiber grating bending sensors in laminated composite structures," Proc. SPIE 3330, 284-292 (1998).

15. D. Kim and J. Kang, "Sagnac loop interferometer based on polarization maintaining photonic crystal fiber with reduced temperature sensitivity," Opt. Express 12, 4490-4495 (2004).

16. A. Michie, J. Canning, K. Lyytikäinen, M. Åslund, and J. Digweed, "Temperature independent highly birefringent photonic crystal fiber," Opt. Express 12, 5160-5165 (2004).

17. T. Ritari, H. Ludvigsen, M. Wegmuller, M. Legré, N. Gisin, J. Folkenberg, and M. Nielsen, "Experimental study of polarization properties of highly birefringent photonic crystal fibers," Opt. Express 12, 5931-5939 (2004). 